\title{
Roles of Neutrophil/Lymphocyte and Platelet/Lymphocyte Ratios in the Early Diagnosis of Malignant Ovarian Masses
}

\author{
Mem Arjen Yildirim, Kerem Doga Seckin*, Cihan Togrul, Eralp Baser, Mehmet \\ Fatih Karsli, Tayfun Gungor, Hacer Cavidan Gulerman
}

\begin{abstract}
Background: The present study aimed to investigate the utility and importance of the various parameters of complete blood count panel for benign-malignant differentiation of adnexal masses. Materials and Methods: This retrospective study involved 316 patients with documented benign and 253 patients with malignant adnexal masses who underwent primary surgical treatment at a tertiary referral center. Prior to the study, all benign and malignant cases were compared within their own groups and then the benign and malignant cases were compared to each other. For all cases, cut-off, sensitivity, specificity, positive predictive value (PPV) and negative predictive value (NPV) were calculated for the neutrophil lymphocyte ratio (NLR), platelet lymphocyte ratio (PLR), neutrophil, lymphocyte, platelet and CA-125 parameters, and the results were compared in regards to the groups. Results: NLR, PLR, neutrophil, CA-125, and platelet values were higher in the malignant compared to the benign cases $(p<0.01)$. The lymphocyte value was lower in the malignant cases $(p<0.01)$. No significant differences were found for basophils and eosinophils $(p>0.05)$. For CA-125, the sensitivity, specificity, PPV and NPV for all cases were $78 \%, 62 \%, 62 \%$ and $78 \%$, respectively. For NLR, they were $65.6 \%, 72.1 \%, 65.3 \%$, and $\mathbf{7 2 . 3 \%}$, and for PLR, $48 \%, 81 \%, 67 \%$, and $66 \%$. Additionally, the sensitivity and specificity were $55 \%$ and $77 \%$ for CA-125, 66\% and $58 \%$ for NLR, and $61 \%$ and $58 \%$ for PLR in early malignant cases. Conclusions: NLR and PLR appear to be useful methods that can be applied together with CA-125 due to the relatively high sensitivity values for the malign-benign differentiation of ovarian masses. Although the specificity of these parameters is lower than CA-125, especially in cases with early malignant ovarian pathology, their sensitivity being higher is promising for the early diagnosis of ovarian cancer. It can be used to detect ovarian malignancies in the early stages, and it will increase the treatment options and improve survival rates.
\end{abstract}

Keywords: Neutrophil lymphocyte ratio - platelet lymphocyte ratio - ovarian mass - CA-125

Asian Pac J Cancer Prev, 15 (16), 6881-6885

\section{Introduction}

Ovarian cancer is the second most common gynecologic cancer, but it is also the gynecologic cancer with the highest mortality rate across the world (Landis et al., 1998). This is because the early disease is usually asymptomatic and most of the detected cases are already in an advanced stage at the time of diagnosis. Approximately $70 \%$ of the women with ovarian cancer are diagnosed in stages 3 and 4; however, 5-year survival is only $40 \%$ (Jemal et al., 2009). Nevertheless, when the disease is detected in the early stagea, it has a significantly better prognosis and a 5-year survival rate approaching 90\% (Pauler et al., 2001).

There are several markers studied in the early detection of ovarian cancer in the preoperative period such as serum CA-125, soluble cytokeratin fragments, serum human kallikrein, serum cytokines, vascular endothelial growth factor (VEGF) and plasma d-dimer (Gadducci et al., 2009, Li et al., 2012). CA-125 is the most useful and common molecular marker for diagnosing ovarian cancer (Arun-Muthuvel and Jaya, 2014). Today, the most commonly used combination is that in which CA-125 and imaging methods are used together (Ashrafgangooei and Rezaeezadeh, 2011). However, this combination is not sufficient for early diagnosis due to the low sensitivity of CA-125 and the cost of imaging methods (Bast et al., 2005). Therefore, development a new marker to increase the early detection rate is necessary. Hematological, inflammatory, or immunological markers that are studied through advanced technology combined with CA-125 may improve diagnostic sensitivity (Bast et al., 1998). Some of the parameters from the complete blood count (CBC) panel have been suggested for use in the diagnosis and also predicting the prognosis (Ueno et al., 2007). It was found that neutrophils increased and the lymphocyte count

${ }^{\prime}$ Obstetrics and Gynecology Department of Zekai Tahir Burak Women's Health and Research Hospital, Ankara, Turkey *For correspondence:doga_seckin@hotmail.com 
relatively decreased as a result of systemic inflammatory response (Jilma et al., 1999). Additionally, the current thrombocytosis tumor aggressiveness was correlated with rapid prognoses and high recurrence rates (Li et al., 2004). NLR (neutrophil/lymphocyte ratio) was increased in the epithelial ovarian cancers and correlated with adverse clinical outcomes (Cho et al., 2009).

The present study aims to detect the value of blood cell counts, as well as PLR and NLR parameters from the $\mathrm{CBC}$ panel in the early detection of ovarian cancer.

\section{Materials and Methods}

This retrospective study involved 569 patients with documented benign and malignant ovarian pathologies who underwent surgery in the gynecology, infertility, and gynecologic oncology departments between January 2006 and December 2011 at Zekai Tahir Burak Women's Health Training and Research Hospital in Ankara, Turkey. Ethical and scientific approvals were obtained from the institutional review board. Data regarding age, parity, menopausal status, the size and laterality of the masses of the patients and the dates of the operation were established. The CBC and tumor markers obtained just prior to the operation dates were recorded. Among the CBC profile, white blood cells, neutrophils, lymphocytes, basophils, and platelets were recorded separately and the platelet/ lymphocyte (PLR) and neutrophil/lymphocyte (NLR) ratios were calculated individually. CA-125 was recorded as the primary tumor marker. Patients with increased white blood cell count that might indicate a pre-existing infection were excluded from the study.

The pathology results of the patients were examined; the histological diagnoses of the benign cases, and stages and grades of the malignant cases in addition to the histological diagnoses were recorded. Only the patients with the pathology of ovarian origin were included in the study. During this selection, cases with malignant and benign ovarian pathology, epithelial and non-epithelial or endometrioma and mature cystic teratoma were included altogether in the study. Additionally, the patients who were operated under the pre- existing diagnoses of ovarian mass or adnexal mass, however, had diagnoses such as paratubal cyst, paraovarian cyst, intraligamentary myoma, and tuboovarian abscess as a pathology result were excluded from the study. The patients were separated into two main groups as malignant and benign, and then subgroups were formed after the comparison with regards to the parameters. The benign cases were compared as endometrioma and other benign cases. The malignant cases were compared among themselves as epithelial and non-epithelial, and also the malignant cases were compared by dividing the groups into subgroups as early and advanced stages. The cut-off value for CA-125 was $35 \mathrm{IU} / \mathrm{ml}$. For other parameters, individual cut-off values were calculated. Additionally, sensitivity, specificity, positive predictive values, and negative predictive values were calculated for each parameter.

\section{Statistical analysis}

The statistical assessment of the data was performed using Statistical Package for Social Sciences (SPSS) for Windows 15.0 package. In order to establish whether the distribution was normal, Kolmogorov-Smirnov and Shapiro-Wilk tests were applied prior to each comparison. Since the groups were not compatible with the normal distribution, Mann Whitney-U and two-sample Kolmogorov tests were used. These two tests were used to compare the CA-125, neutrophil, lymphocyte, monocyte, eosinophil, basophil, platelet, PLR, and NLR values. An individual ROC curve was applied for each parameter. The cut-off values, sensitivity, specificity, PPD, and NPD values were calculated for all cases; and calculated separately as malignant early stage, malignant advanced stage, premenopausal, and menopausal in case of subgroups. In terms of the results achieved, $\mathrm{p}<0.05$ was statistically considered to be a significant difference between the groups at a confidence interval of $95 \%$.

\section{Results}

The cases involved in the present study include 569 patients in total, 253 of whom were malignant and operated in the oncology clinic, and 316 were benign and operated in the gynecology and infertility clinics. The mean age of the benign cases was 35,20 (95\% CI $33,99-36-41)$ compared to 50,77 (95\% CI, 49,11-52,43) for the malignant patients. When these benign cases were compared among themselves as endometrioma and other benign cases; and the sensitivity value for CA-125 was calculated as $75 \%$, whereas the specificity value was calculated as $82.2 \%$. Although the sensitivity of NLR

Table 1. Diagnostic values of the StudyParameters in Discriminating Various Adnexal Masses Discriminating Endometriomas from other Benign Lesions

\begin{tabular}{|c|c|c|c|c|c|c|}
\hline & AUC & 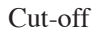 & & & & ${ }^{e} \mathrm{NP}$ \\
\hline \multicolumn{2}{|l|}{ CA-125 } & 35 & 0.75 & 0.8229 & 0.7322 & 0.8359 \\
\hline bNLR & 0.593 & 16,863 & 0.79 & 0.3697 & 0.4336 & 0.7319 \\
\hline${ }^{\mathrm{c} P L R}$ & 0.621 & 115.65 & 0.758 & 0.484 & 0.4870 & 0.7560 \\
\hline Neutrophil & 0.701 & 5,395 & 0.3064 & 0.7968 & 0.4935 & 0.6416 \\
\hline Lymphocyte & 0.593 & 1,995 & 0.5 & 0.64 & 0.4732 & 0.6648 \\
\hline Platelet & 0.56 & 239.5 & 0.7419 & 0.3958 & 0.4423 & 0.6294 \\
\hline \multicolumn{7}{|c|}{ Discriminating all malignant cases from benign cases } \\
\hline CA-125 & & 35 & 0.7865 & 0.6297 & 0.6297 & 0.7865 \\
\hline bNLR & 0.737 & 24,114 & 0.6561 & 0.7215 & 0.6535 & 0.7238 \\
\hline${ }^{\mathrm{c}} \mathrm{PLR}$ & 0.684 & 173.76 & 0.4842 & 0.8196 & 0.6777 & 0.6632 \\
\hline Neutrophil & 0.701 & 4.46 & 0.7312 & 0.577 & 0.5781 & 0.7269 \\
\hline Lymphocyte & 0.609 & 1.33 & 0.2332 & 0.9398 & 0.7662 & 0.6048 \\
\hline Platelet & 0.645 & 359.5 & 0.3438 & 0.8924 & 0.719 & 0.6294 \\
\hline \multicolumn{7}{|c|}{ Discriminating early stage malignant cases from benign cases } \\
\hline CA-125 & & 35 & 0.5576 & 0.7743 & 0.3372 & 0.8122 \\
\hline bNLR & 0.656 & 2.11 & 0.667 & 0.589 & 0.3434 & 0.8454 \\
\hline 'PLR & 0.594 & 132.61 & 0.6153 & 0.5854 & 0.3457 & 0.7859 \\
\hline Neutrophil & 0.649 & 4.46 & 0.6826 & 0.5727 & 0.3446 & 0.8457 \\
\hline Lymphocyte & 0.547 & 2.46 & 0.8076 & 0.2943 & 0.2736 & 0.8230 \\
\hline Platelet & 0.546 & 333.5 & 0.2884 & 0.8259 & 0.3529 & 0.7791 \\
\hline \multicolumn{7}{|c|}{ Discriminating advanced stage malignant cases from benign cases } \\
\hline CA-125 & & 35 & 0.9562 & 0.6357 & 0.5346 & 0.9707 \\
\hline bNLR & 0.777 & 24,114 & 0.7445 & 0.7215 & 0.5368 & 0.8669 \\
\hline${ }^{\mathrm{c}} \mathrm{PLR}$ & $0.74 \quad 1$ & $, 856,832$ & 0.5579 & 0.8575 & 0.6311 & 0.8162 \\
\hline Neutrophil & 0.732 & 4,715 & 0.6934 & 0.6487 & 0.4611 & 0.8299 \\
\hline Lymphocyte & 0.651 & 1.33 & 0.30430 & 0.9398 & 0.6885 & 0.7557 \\
\hline Platelet & 0.8162 & 359.5 & 0.4347 & 0.8924 & 0.6382 & 0.7833 \\
\hline
\end{tabular}

*aAUC: Area under curve, ${ }^{b}$ NLR: Neutrophil Lymphocyte Ratio, ${ }^{\text {} P L R: ~ P l a t e l e t ~}$ Lymphocyte Ratio, ${ }^{\mathrm{d} P P V}$ : Positive Predictive Value, ${ }^{\mathrm{e}} \mathrm{NPV}$ : Negative Predictive Value 
was higher than CA-125, its specificity value was lower than CA-125 (36.9\%). The sensitivity of PLR was equal to the value of CA-125, while its specificity was higher than NLR. The test with the highest PPV and NPV values was CA-125.

When the malignant cases were compared among themselves as epithelial and non-epithelial, no statistically significant difference was found in NLR, PLR, lymphocyte, monocyte, eosinophil, and basophil parameters ( $p>0.05$ ), whereas there was a significant difference between the platelets and neutrophils $(\mathrm{p}<0.01)$ (Table 1). When the malignant cases were compared to the benign cases, no difference was observed in neutrophil and basophil distributions between the patients $(p>0.05)$, whereas there was a difference in platelet, neutrophil, lymphocyte, monocyte, NLR, and PLR values between the malignant and benign patients $(\mathrm{p}<0.01)$. Within the parameters displaying differences between the two groups, only the lymphocyte values were higher in the benign cases compared to the malignant cases, whereas other parameters displaying differences between the two groups were higher in the malignant cases compared to the benign cases. ROC curves, specificity, sensitivity, NPV, and PPV were individually calculated for the NLR and PLR values, which were the subject of the present study (Figure 1AB). For the hematological parameters of the malignant and benign cases, CA-125 had the highest sensitivity (78\%) and the lymphocyte had the highest specificity among the sensitivity, specificity, PPV, and NPV values. The lymphocyte was found to be the parameter with the highest PPD value (76\%) and CA-125 was found to have the highest NPD value (78\%) (Table 1).

One hundred four early-stage cases (Stages 1 and 2) and 149 advanced-stage cases (Stages 3 and 4) with malignant ovarian pathology were compared to the cases with benign pathology, and a significant difference was found in NLR, PLR, CA-125, platelet, neutrophil, and lymphocyte values between the groups $(\mathrm{p}<0.01)$. The

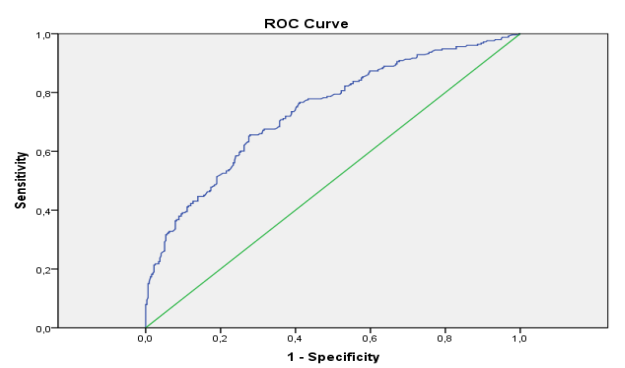

Figure 1A. ROC Curve for Neutrophil Lymphocyte Ratio (NLR)

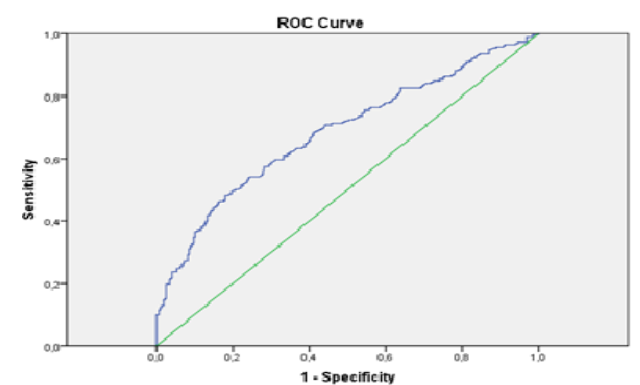

Figure 1B. ROC Curve for Platelet Lymphocyte Ratio (PLR) highest sensitivity was observed with the lymphocyte in the early-stage cases, whereas the highest specificity was found with the platelet values (Table 1). The highest sensitivity was observed with CA-125 when the advancedstage cases were compared, whereas the highest specificity was found with the lymphocyte values (Table 1).

\section{Discussion}

Ovarian cancer has a fatal course, due to the fact that it is usually diagnosed in the advanced stage. Therefore, the focus is placed on the early detection of the disease and since the early-stage patients are generally asymptomatic, the early detection of ovarian cancer and research in this field has gained significance (Benedet et al., 2000, Bian et al., 2013, Winarto et al., 2014, ArunMuthuvel and Jaya, 2014). Chronic inflammation has been identified as a risk factor in the cancers of epithelial origin (Jaiswal et al., 2001; Brower, 2005). In addition to infection, chronic inflammation may develop due to autoimmune diseases, malignant and benign tumors, and the pathologies that result in infiltration in specific parts of the body. Thrombocytosis was observed in tumoral formations; the lymphocyte count was relatively reduced and the neutrophil count increased. It is known that inflammation contributes to the development and progression of various cancers. Examples may include lung, breast, gastrointestinal, ovarian, prostate, skin, and liver cancers (Ardies, 2003; Altinoz and Korkmaz, 2004; Bartsch and Nair, 2004; Van der Auwera et al., 2004; Wang et al., 2004; Hussein and Ahmed, 2005) (Dirican et al., 2013; Karaman et al., 2013; Unal et al., 2013) These inflammation markers have been studied in gynecological cancers and some gynecological benign diseases (Guzel et al., 2014). The study by Suh et al. (2012) reported that the value of NLR, which was also the subject of the present study, being above 1.97 was more effective than CA-125 in the prediction of the lymph node metastasis in the endometrioid endometrial carcinomas (Suh et al., 2012). In another study by Lee et al. (2012), NLR was studied prior to the treatment in cervical cancer and it was shown that this value might be used to estimate mortality and recurrence rates (Lee et al., 2012). NLR was found to be more effective than CA-125 in the preoperative differentiation of uterine sarcomas from the other uterine diseases (myoma uteri, adenomyosis), recurrences and the prediction of prognosis (Kim et al., 2010). The increased platelet count in ovarian tumors, especially in studies about epithelial ovarian cancers, was found to be associated with poor prognosis and low survival rates in the advanced epithelial ovarian cancers that were treated with surgery and chemotherapy (Crasta et al., 2010; Kuyumcuoglu et al., 2010; Lee et al., 2011). In addition to the platelet count, NLR and PLR were also found to be associated with poor prognosis and low survival rates (Asher et al., 2011; Thavaramara et al., 2011). The study conducted by Cho et al. (2008) investigated the value of NLR in the differentiation of endometrioma from the other benign cases (Cho et al., 2008). The sensitivity and specificity for NLR were $59.7 \%$ and $60.1 \%$, respectively. When the same groups were compared in the present 
study, the sensitivity value was higher $(79 \%)$, whereas the specificity value was $36 \%$. It was established that the highest sensitivity was with the neutrophil (68.4\%); however, on the contrary, the neutrophil parameter had the lowest sensitivity in the present study (30.6\%). When these results were analyzed, NLR was found to have a better sensitivity compared to CA-125 (75\%) in differentiating the cases with endometrioma from the other benign cases. The sensitivity of PLR $(75.8 \%)$ was equal to CA-125. These parameters can be used as a tool for scanning, but do not appear to be appropriate for use in the differential diagnosis due to their low specificity values. Consequently, CA-125 is still considered as the most ideal parameter in the diagnosis and scanning of the endometriomas; nevertheless, other parameters have the potential to be used as a tool for scanning alone or in combination with CA-125 (Winarto et al., 2014). Hanbyoul et al. compared the NLR values between the early ovarian cancers and benign cases. They established the sensitivity, specificity, and cut-off values of NLR separately for all malignant cases and early malignant cases (Cho et al., 2009). Neutrophils and monocytes were higher, and lymphocytes, basophils, and eosinophils were lower in malignant patients compared to the benign patients. In the present study, the neutrophil, monocyte and platelet counts were higher and the lymphocyte count was lower in malignant patients. When all cases were evaluated, CA-125 had the highest sensitivity with $78.6 \%$ among the parameters studied. CA-125 was the optimal parameter for the scanning test in the present study. However, NLR, which had a sensitivity of $65.6 \%$, and neutrophils, which had a sensitivity of $73.1 \%$, appeared to be useful parameters for the scanning test. Additionally, NLR had a higher specificity value with $72 \%$ compared to CA-125. Among the other parameters with high specificity values, this value was found as $93.9 \%$ for lymphocytes, $89.2 \%$ for platelets, and $81.9 \%$ for PLR, respectively. Therefore, platelets, lymphocytes and PLR stand out as useful parameters for the differential diagnosis. The sensitivity of CA-125 was $78 \%$ for all cases, whereas this rate was $55 \%$ in the early-stage patients. The value of the highest specificity was found for platelets as $82 \%$. Consequently, when the early-stage patients were considered, the scanning test performance of lymphocytes, neutrophils, NLR, and PLR was higher than CA-125. However, the low specificity value of the lymphocytes (29\%) may increase the false positivity rate. The fact that no statistical significant difference was found for NLR and PLR between the advanced and early malignant cases suggests that these parameters can be used to predict prognoses and recurrences. When all cases with malignant ovarian pathology were compared with all cases with benign ovarian pathology, CA-125 came to the forefront as the most important scanning test, whereas NLR and neutrophils might be used alone or in combination with this test. However, when the early-stage patients were considered, the sensitivity of NLR, PLR, neutrophil, and lymphocyte parameters were higher than CA-125. Therefore, these parameters may provide great benefits for detecting early-stage patients, which is the mainly targeted issue. The cases with a NLR cut-off value above 2.11 among the early malignant cases having similar distribution and values with the cases that have a CA-125 value $<35$ and $>35$ may indicate that NLR can be used independently from CA-125 in scanning the early-stage cases. However, the PLR values of the cases with a PLR cut-off value above 132, six were found to be higher in the cases with a CA- 125 value $>35$ compared to the cases with a CA-125 value $<35$. This suggests that PLR may be more effective in scanning the early-stage malignant cases when used in combination with CA-125. In consequence, use of these parameters either alone or together may contribute to detect malignant ovarian pathologies in the early stages, to increase the available treatment options and to improve the survival rates. Nevertheless, the efficacy of these values in regards to the parameters used in the present study should be supported by the studies involving more cases.

\section{References}

Altinoz MA, Korkmaz R (2004). NF-kappaB, macrophage migration inhibitory factor and cyclooxygenase-inhibitions as likely mechanisms behind the acetaminophen- and NSAID-prevention of the ovarian cancer. Neoplasma, 51, 239-47.

Ardies CM (2003). Inflammation as cause for scar cancers of the lung. Integr Cancer Ther, 2, 238-46.

Arun-Muthuvel V, Jaya V (2014). Pre-operative evaluation of ovarian tumors by risk of malignancy index, CA125 and ultrasound. Asian Pac J Cancer Prev, 15, 2929-32.

Asher V, Lee J, Innamaa A, Bali A (2011). Preoperative platelet lymphocyte ratio as an independent prognostic marker in ovarian cancer. Clin Transl Oncol, 13, 499-503.

Ashrafgangooei T, Rezaeezadeh M (2011). Risk of malignancy index in preoperative evaluation of pelvic masses. Asian Pac J Cancer Prev, 12, 1727-30.

Bartsch H, Nair J (2004). Oxidative stress and lipid peroxidationderived DNA-lesions in inflammation driven carcinogenesis. Cancer Detect Prev, 28, 385-91.

Bast RC, Jr. Badgwell D, Lu Z, et al (2005). New tumor markers: CA125 and beyond. Int J Gynecol Cancer, 15, 274-81.

Bast RC, Jr Xu FJ, Yu YH, et al (1998). CA 125: the past and the future. Int J Biol Markers, 13, 179-87.

Benedet JL, Bender H, Jones H, Ngan HY, Pecorelli S (2000). FIGO staging classifications and clinical practice guidelines in the management of gynecologic cancers. FIGO Committee on Gynecologic Oncology. Int J Gynaecol Obstet, 70, 209-62.

Bian J, Li B, Kou XJ, Liu TZ, Ming L (2013). Clinical significance of combined detection of serum tumor markers in diagnosis of patients with ovarian cancer. Asian Pac J Cancer Prev, 14, 6241-3.

Brower V (2005). Feeding the flame: new research adds to role of inflammation in cancer development. J Natl Cancer Inst, 97, 251-3.

Cho H, Hur HW, Kim SW, et al (2009). Pre-treatment neutrophil to lymphocyte ratio is elevated in epithelial ovarian cancer and predicts survival after treatment. Cancer Immunol Immunother, 58, 15-23.

Cho S, Cho H, Nam A, et al (2008). Neutrophil-to-lymphocyte ratio as an adjunct to CA-125 for the diagnosis of endometriosis. Fertil Steril, 90, 2073-9.

Crasta JA, Premlatha TS, Krishnan SM, VallikadE, Rameshkumar K (2010). Significance of preoperative thrombocytosis in epithelial ovarian cancer. Indian J Pathol Microbiol, 53, 54-6.

Dirican A, Kucukzeybek Y, Erten C, et al (2013). Prognostic 
and predictive value of hematologic parameters in patients with metastatic renal cell carcinoma: second line sunitinib treatment following IFN-alpha. Asian Pac J Cancer Prev, 14, 2101-5.

Gadducci A, Cosio S, Tana R, Genazzani AR (2009). Serum and tissue biomarkers as predictive and prognostic variables in epithelial ovarian cancer. Crit Rev Oncol Hematol, 69, 12-27.

Guzel AI, Kokanali MK, Erkilinc S, et al (2014). Predictive role of the neutrophil lymphocyte ratio for invasion with gestational trophoblastic disease. Asian Pac J Cancer Prev, 15, 4203-06.

Hussein MR, Ahmed RA (2005). Analysis of the mononuclear inflammatory cell infiltrate in the non-tumorigenic, pretumorigenic and tumorigenic keratinocytic hyperproliferative lesions of the skin. Cancer Biol Ther, 4, 819-21.

Jaiswal M, Larusso NF, Gores GJ (2001). Nitric oxide in gastrointestinal epithelial cell carcinogenesis: linking inflammation to oncogenesis. Am J Physiol Gastrointest Liver Physiol, 281, 626-34.

Jemal A, Siegel R, Ward E, et al (2009). Cancer statistics, 2009. CA Cancer J Clin, 59, 225-49.

Jilma B, Blann A, Pernerstorfer T, et al (1999). Regulation of adhesion molecules during human endotoxemia. No acute effects of aspirin. Am J Respir Crit Care Med, 159, 857-63.

Karaman H, Karaman A, Erden A, et al (2013) Relationship between colonic polyp type and the neutrophil/ lymphocyte ratio as a biomarker. Asian Pac J Cancer Prev, 14, 3159-61.

Kim HS, Han KH, Chung HH, et al (2010). Neutrophil to lymphocyte ratio for preoperative diagnosis of uterine sarcomas: a case-matched comparison. Eur J Surg Oncol, 36, 691-8.

Kuyumcuoglu U, Guzel AI, Celik Y, Erdemoglu M (2010). The association of preoperative thrombocytosis with prognostic factors in malign ovarian tumor. Eur J Gynaecol Oncol, 31, 514-6.

Landis SH, Murray T, Bolden S, Wingo PA (1998). Cancer statistics, 1998. CA Cancer J Clin, 48, 6-29.

Lee M, Kim SW, Nam EJ, et al (2011). The impact of pretreatment thrombocytosis, persistent thrombocytosis after adjuvant chemotherapy in patients with advanced epithelial ovarian cancer. Gynecol Oncol, 122, 238-41.

Lee YY, Choi CH, Kim HJ, et al (2012). Pretreatment neutrophil:lymphocyte ratio as a prognostic factor in cervical carcinoma. Anticancer Res, 32, 1555-61.

Li AJ, Madden AC, Cass I, et al (2004). The prognostic significance of thrombocytosis in epithelial ovarian carcinoma. Gynecol Oncol, 92, 211-4.

$\mathrm{Li} \mathrm{L}, \mathrm{Xu}$ Y, Yu CX (2012). Proteomic analysis of serum of women with elevated $\mathrm{Ca}-125$ to differentiate malignant from benign ovarian tumors. Asian Pac J Cancer Prev, 13, 3265-70.

Pauler DK, Menon U, Mcintosh M, et al (2001). Factors influencing serum CA125II levels in healthy postmenopausal women. Cancer Epidemiol Biomarkers Prev, 10, 489-93.

Suh DH, Kim HS, Chung HH, et al (2012). Pre-operative systemic inflammatory response markers in predicting lymph node metastasis in endometrioid endometrial adenocarcinoma. Eur J Obstet Gynecol Reprod Biol, 162, 206-10.

Thavaramara T, Phaloprakarn C, Tangjitgamol S, Manusirivithaya S (2011). Role of neutrophil to lymphocyte ratio as a prognostic indicator for epithelial ovarian cancer. $\mathrm{J} \mathrm{Med}$ Assoc Thai, 94, 871-7.

Ueno H, Hawrylowicz CM, Banchereau J (2007). Immunological intervention in human diseases. J Transl Med, 5, 59.

Unal D, Eroglu C, Kurtul N, Oguz A, Tasdemir A (2013). Are neutrophil/lymphocyte and platelet/lymphocyte rates in patients with non-small cell lung cancer associated with treatment response and prognosis? Asian Pac J Cancer Prev, 14, 5237-42.

Van Der Auwera I, Van Laere SJ, Van Den Eynden GG, et al (2004). Increased angiogenesis and lymphangiogenesis in inflammatory versus noninflammatory breast cancer by real-time reverse transcriptase-PCR gene expression quantification. Clin Cancer Res, 10, 7965-71.

Wang W, Bergh A, Damber JE (2004). Chronic inflammation in benign prostate hyperplasia is associated with focal upregulation of cyclooxygenase-2, Bcl-2, and cell proliferation in the glandular epithelium. Prostate, 61, 60-72.

Winarto H, Laihad BJ, Nuranna L (2014). Modification of cutoff values for HE4, CA125, the risk of malignancy index and the risk of malignancy algorithm for ovarian cancer detection in Jakarta, Indonesia. Asian Pac J Cancer Prev, 15, 1949-53. 